\title{
Les tensions de rôle chez les apprenti·e•s pour caractériser la qualité perçue de la formation professionnelle en alternance et ses conséquences
}

\section{Apprentices' role stress to characterize the perceived quality of dual vocational education and training and its consequences}

Matilde Wenger ${ }^{\mathbf{1}}$, Junior Researcher, Institut fédéral des hautes études en formation professionnelle (IFFP), Renens, Suisse

Florinda Sauli ${ }^{2}$, Junior Researcher, Institut fédéral des hautes études en formation professionnelle (IFFP), Renens, Suisse

Jean-Louis Berger ${ }^{3}$, Professeur ordinaire, Université de Fribourg, Suisse

Titre courant : Tensions de rôle chez les apprenti·e $\cdot \mathrm{s}$

Résumé : Dans le contexte de la formation professionnelle initiale (FPI) en alternance, une étude suisse romande s'est penchée sur les perceptions de qualité d'une telle formation par des apprenti·e.s. Une partie des caractéristiques de la qualité perçue en FPI est notamment liée aux divers rôles que les apprenti-e.s assument à l'école et en entreprise et aux conséquences que ces rôles peuvent avoir sur leur vécu en apprentissage.

À partir des réponses d'apprenti $\cdot e \cdot s$ en alternance $(N=336)$, une analyse factorielle exploratoire a permis de distinguer quatre dimensions faisant référence à des tensions de rôle ; des analyses de cheminement ont montré que la perception de tensions de rôle a un lien négatif avec le sentiment d'auto-efficacité, l'engagement et la satisfaction. Ces résultats illustrent ainsi que différentes tensions de rôle peuvent être associées au vécu des apprenti·e·s en FPI, et que ces tensions ont des effets néfastes sur le bien-être des personnes en formation. La discussion propose des pistes pour clarifier les rôles de ces dernier·ère.s.

Abstract: In the context of dual initial vocational education and training (IVET), a study in French-speaking Switzerland looked at apprentices' perceptions of the quality of such training. Some of the perceived quality characteristics of IVET are related to the various roles that apprentices assume at school and in the training company and the consequences that these roles may have on their apprenticeship experience.

On the basis of dual apprentices' responses ( $\mathrm{N}=336)$, an exploratory factor analysis has identified four dimensions referring to role tensions; path analyses showed that the perception of role tensions was negatively related to self-efficacy, commitment and satisfaction. These results illustrate that different role stress can be associated with the IVET apprentices' experience, and that this stress has negative effects on the apprentices' well-being. The discussion part proposes to clarify the latter's roles.

Mots-clés : tensions de rôle, qualité de la formation professionnelle, apprentissage en alternance, formation professionnelle suisse

Keywords: role stress, VET quality, dual apprenticeship, Swiss VET

\footnotetext{
${ }^{1}$ Thèmes de recherche : Qualité de la formation professionnelle, tensions de rôle. matilde.wenger@iffp.swiss

2 Thèmes de recherche: Qualité de la formation professionnelle, connexion entre lieux de formation. florinda.sauli@iffp.swiss

3 Thèmes de recherche: Formation professionnelle, motivation, apprentissage autorégulé. jeanlouis.berger@unifr.ch
} 


\section{Introduction}

Entreprendre un apprentissage en alternance (désigné formation duale en Suisse) implique pour les apprenti-e.s d'accéder au monde du travail tout en continuant à fréquenter l'école. Les enjeux en termes de qualité d'une telle formation sont par conséquent multiples : il s'agit notamment de garantir des apprentissages scolaires en accord avec la formation pratique en entreprise (Berger et al., 2019b ; Guile \& Young, 2003), ou encore de pouvoir compter sur des enseignant·e.s et des personnes formatrices compétent·e.s (Berger et al., 2019a; Smith \& Yasukawa, 2017). À ces éléments rendant principalement compte d'aspects institutionnels et formels de la formation professionnelle (FP) en alternance s'ajoute l'aspect subjectif du vécu des apprenti-e.s, qui s'avère central dans la définition de la qualité en formation professionnelle (Gross et al., 2020 ; Stalder \& Carigiet Reinhard, 2014). Il s'agit notamment de tensions que les apprenti.e.s peuvent ressentir quant $\mathrm{au}(\mathrm{x})$ rôle(s) qu'elles et ils assument durant leur formation en école et en entreprise et qui constituent pour partie leur conception de la qualité (Alves et al., 2010b ; Wenger, sous presse).

Partant, les perceptions des apprenti-e.s, par rapport à la qualité de leur formation, ont été investiguées par le biais d'un questionnaire comprenant des questions sur les tensions de rôle en tant que caractéristiques de la qualité perçue. Ces tensions ont ensuite été analysées en lien avec certaines conséquences d'une formation de plus ou moins bonne qualité : le sentiment d'efficacité personnelle, l'engagement, la satisfaction et l'intention de résilier le contrat d'apprentissage.

Après une brève présentation du système de FP dual suisse - en lien notamment avec la notion de qualité et des diverses formes de tensions de rôle -, nous présentons une étude ayant pour but d'investiguer les tensions de rôle en tant que caractéristiques de la qualité perçue chez les apprenti·e·s ainsi que certaines résultantes de cette qualité.

\subsection{Le système de FP en alternance helvétique face aux défis de la qualité}

En Suisse, deux jeunes sur trois suivent une formation professionnelle initiale (FPI) (Office fédéral de la statistique [OFS], 2017), soit en modalité duale (90\% des apprenti-e.s), soit en école à plein temps $(10 \%$ des apprenti-e.s ; Secrétariat d'État à la formation, à la recherche et à l'innovation [SEFRI], 2018). En ce qui concerne la FP en alternance, celle-ci combine une partie théorique en école professionnelle (un à deux jours par semaine) et une partie de formation en entreprise (à hauteur de quatre jours par semaine dans la plupart des métiers). Ce double système de formation permet aux apprenti-e.s d'apprendre un métier en se concentrant tant sur les connaissances procédurales que sur les connaissances déclaratives spécifiques à ce métier. La formation vise également à dispenser des connaissances dites de culture générale. Ainsi, un tel système met l'accent sur les entreprises comme principales actrices de la formation, en confrontant les jeunes dès le début de leur formation à des dynamiques et phénomènes propres au marché du travail (Masdonati et al., 2007).

Le modèle dual helvétique, vanté à l'étranger, est considéré comme étant d'excellente qualité, permettant notamment un accès facilité au marché du travail (Dubs, 2006) et par conséquent un faible taux de chômage des jeunes (OFS, 2019). Partant, la qualité dans un tel système de formation doit pouvoir être garantie, ce qui est formalisé par plusieurs textes, tels que, par exemple, la loi fédérale sur la formation professionnelle du 13 décembre 2002. Ces derniers précisent la volonté de développer et surveiller la qualité du système, mais ne définissent pas la qualité, ni mentionnent concrètement comment celle-ci peut être consolidée, cette tâche semblant appartenir aux institutions du terrain (cantons, écoles professionnelles et entreprises formatrices). Par ailleurs, la qualité du système de FP a tendance à être assimilée à 
l'employabilité élevée chez les jeunes, ce qui est certes un bon indicateur, mais pas l'unique facteur à jouer un rôle dans la définition de cette qualité. Finalement, l'évaluation de la qualité en FP en Suisse n'est pas systématiquement exigée par les institutions ; le cas échéant, elle se réalise, entre autres, par le biais d'un instrument présentant exclusivement des critères observables concernant l'entreprise (Centre suisse de services Formation professionnelle [CSFO], 2012). Dans ce contexte, la perspective des apprenti·e $\cdot \mathbf{s}-$ bénéficiaires ultimes de ce système - ne rentre pas officiellement en ligne de compte dans les mesures de développement de la qualité en FP.

Le projet dans lequel s'inscrit cette étude vise par conséquent à mieux appréhender la notion de qualité en FP en s'intéressant aux perceptions de ses principaux acteurs du terrain: les personnes formatrices en entreprise, les enseignant-e.s en école professionnelle et les apprenti·e.s en FPI duale dans quatre champs professionnels (coiffure et soins de beauté, construction, commerce de détail et employé.e.s de commerce ${ }^{4}$ ) en Suisse romande.

Le concept de qualité, bien que largement utilisé dans de nombreux contextes, n'a pas de définition univoque, ce qui lui confère des connotations plurielles et relatives (Bouchard \& Plante, 2002; Wittek \& Kvernbekk, 2011). Ainsi, le premier aspect à considérer dans la définition de la qualité en FP est la pluralité de points de vue sur celle-ci (Griffin, 2017). Ces points de vue peuvent être exprimés par les enseignant.e.s, les personnes formatrices en entreprise, mais aussi les institutions qui participent à diriger la FP (cantons, confédération, organisations du travail) et, finalement, ses principales et principaux «protagonistes »: les apprenti·e.s. La qualité d'une FP peut donc être définie comme la conception subjective d'un idéal vers lequel devrait tendre la formation. Cet idéal peut différer selon les acteurs de la FP et selon le champ professionnel concerné (Berger et al., 2020 ; Griffin, 2017). La notion de qualité est fondée en particulier sur des jugements d'adéquation aux objectifs et d'adéquation des objectifs (Wittek \& Kvernbekk, 2011) par rapport aux attentes et besoins personnels.

Dans la littérature, la problématique de la qualité en FP s'exprime souvent en termes d'évaluation, ce qui a donné naissance à plusieurs instruments permettant de mesurer indirectement par le biais de perceptions (Plante \& Bouchard, 1998) - la qualité (Rauner, 2007 ; Velten \& Schnitzler, 2012). Plusieurs éléments ont été identifiés comme caractérisant une formation de bonne qualité : les compétences des personnes formant les apprenti·e·s à l'école et en entreprise, l'autonomie de ces dernier-ère-s, mais aussi leur stress perçu (Stalder \& Carigiet Reinhard, 2014). En ce sens, parmi les caractéristiques de la qualité, celles liées au vécu des apprenti-e.s occupent une place centrale dans l'évaluation de la qualité d'une formation. Cependant, malgré l'intérêt pour les expériences scolaires et professionnelles des apprenti·e.s du dual, les différents rôles qu'elles et ils assument n'ont pas, à ce jour, été directement étudiés comme caractéristiques de la qualité.

Les analyses présentées dans cette étude ont pour but d'approfondir la perspective des apprenti·e $\cdot \mathrm{s}$ en FPI duale et, plus précisément, leurs perceptions quant à leurs rôles et aux liens existants avec des phénomènes tels que la satisfaction et l'engagement.

\subsection{Apprécier la qualité d'une formation en alternance à partir des tensions de rôle perçues par les apprenti·e $\cdot s$}

Dans le cadre de leur formation en entreprise, les apprenti·e·s n'apprennent pas seulement un métier, mais s'inscrivent également dans un processus de socialisation professionnelle (Dubar, 1992 ; Martineau et al., 2009), soit à l'appropriation et adaptation à leur environnement de

\footnotetext{
${ }^{4}$ Ce métier correspond au travail administratif ou de bureau.
} 
formation. Ce processus lié à l'intégration en entreprise devrait permettre aux apprenti $\cdot e \cdot s$ de développer une identité professionnelle en interaction avec ce que Lave et Wenger (1991) appellent les communautés de pratique : des individus qui partagent les mêmes buts dans leur environnement professionnel et qui, à travers leurs compétences et connaissances, coconstruisent une communauté. Les apprenti.e.s en FP duale intègrent donc une communauté de pratique par le biais de l'entreprise qui les forme et doivent apprendre à en cerner le fonctionnement, notamment par des pratiques de participation au travail telles que des activités ou des interactions avec les collègues (Billett, 2001, 2009).

Outre les enjeux de socialisation dans l'entreprise formatrice et les tensions qui peuvent en découler, les apprenti·e.s font également face à une tension propre à la FP en alternance : la conciliation entre l'école et l'entreprise. Ainsi, les apprenti·e's doivent jongler entre deux univers distincts et il est donc "nécessaire de considérer la position ambiguë, voire même paradoxale de l'apprenti $\cdot e$ (apprenant $\cdot e ~ v s$ travailleur ou travailleuse) » (Losa \& Filliettaz, 2018, p.299). Autrement dit, les apprenti-e.s alternent des moments de socialisation à l'école où elles et ils sont considéré.e. $\mathrm{s}$ comme des élèves - et dans l'entreprise formatrice - où en revanche on les considère comme des travailleur.euse-s. Si un tel statut entre deux peut représenter un atout - de par la possibilité de mettre en commun des apprentissages scolaires et professionnels, «pour les apprenant $\cdot \mathrm{e} \cdot \mathrm{s}$, une telle position entre deux pourrait générer des contradictions [...] étant étudiant·e's et novices qui apprennent et en même temps professionnel-le·s censé·e.s connaître et agir » (Losa \& Filliettaz, 2018, p.304). Dans le cadre de la socialisation des apprenti·e·s, il est par conséquent intéressant d'appréhender les divers rôles qu'elles et ils peuvent assumer.

\subsubsection{Les tensions de rôle}

Lorsqu'un individu est actif dans un contexte professionnel, plusieurs attentes différentes sont formulées à son égard. Elles peuvent venir des collègues, de la clientèle, de la hiérarchie ou de l'individu lui-même et, quand il devient difficile de répondre à l'ensemble de ces attentes de manière satisfaisante, l'individu éprouve des tensions (ou stress en anglais) de rôle (Royal \& Brassard, 2010).

Dans l'approche des tensions de rôle, Rizzo et al. (1970) les repartissent en conflits et ambiguïté. Le premier concept correspond à l'incongruence entre les différentes attentes liées à un rôle (en termes de valeurs, ressources ou exigences). Le concept d'ambiguïté de rôle reflète quant à lui la clarté et la prédictibilité des demandes au sein de l'entreprise. Cette théorie des tensions de rôle a été reprise dans de nombreux travaux, en étudiant notamment les liens entre les divers concepts et leurs effets négatifs (insatisfaction au travail, intention de résilier le contrat de travail, etc. ; Örtqvist \& Wincent, 2006). Un modèle plus récent, que nous présentons ci-après, ajoute le concept de surcharge de rôle (Djabi \& Perrot, 2016 ; Djabi et al., 2019).

Le conflit de rôle est défini par Djabi et Perrot (2016) comme l' " occurrence simultanée d'au moins deux demandes incompatibles relatives au travail demandé » (Djabi \& Perrot, 2016, p.141). Les auteurs distinguent quatre types de conflits de rôle : a) intra-émetteur ; b) interémetteur ; c) inter-rôle ; d) personne-rôle. Le premier type concerne le fait de percevoir des attentes incompatibles venant de la même personne (par exemple un collègue formulant des demandes contradictoires) ; lors d'un conflit inter-émetteur, les attentes incompatibles viennent de personnes différentes (par exemple un patron communique des attentes différentes à celles émises par un collègue) ; le conflit inter-rôle fait référence à la coexistence de plusieurs rôles chez le même individu, rôles auxquels sont associées des demandes incompatibles ; finalement, le conflit personne-rôle apparaît si les attentes liées à un rôle sont en conflit avec les valeurs ou les besoins de l'individu. 
L'ambiguïté de rôle est définie comme la «résultante d'un décalage entre l'information disponible et l'information requise pour l'exercice du rôle par la personne focale » (Djabi \& Perrot, 2016, p.141). Autrement dit, il y a ambiguïté de rôle quand il y a un manque d'informations nécessaires à la réalisation efficace du travail. Il en existe deux formes : a) celle concernant la tâche et $b$ ) celle associée à la dimension socio-émotionnelle. La première fait référence à « un manque d'information concernant les définitions de l'emploi, ses objectifs et les moyens autorisés pour sa mise en œuvre » (Djabi \& Perrot, 2016, p.141), soit par exemple l'étendue des responsabilités de l'individu. La seconde forme se réfère quant à elle à un manque d'information sur la performance de rôle, soit comment l'individu est évalué et les conséquences de cette performance.

La surcharge de rôle est la troisième composante des tensions de rôle et fait référence à des attentes perçues comme excessives en termes de temps ou de ressources (Djabi \& Perrot, 2016 ; Örtqvist \& Wincent, 2006). Dans les travaux plus anciens (Rizzo et al., 1970), ce concept faisait partie des conflits de rôle; ce n'est que dans les dernières années que la surcharge a été considérée comme une composante à part entière. Les méta-analyses effectuées ont en effet permis de démontrer que la surcharge de rôle - considérée distinctement du conflit et de l'ambiguïté - peut avoir un impact spécifique sur plusieurs résultantes professionnelles telles que l'insatisfaction, le manque d'engagement organisationnel ou l'intention de résilier le contrat de travail (Örtqvist \& Wincent, 2006 ; Tubre \& Collins, 2000).

Malgré l'ancrage des tensions de rôle dans le contexte professionnel (Rizzo et al., 1970), seuls quelques travaux se sont penchés sur ces tensions en FP par alternance (Alves et al., 2010a, b ; Perrot, 2005). Ainsi, et au vu du statut « hybride» des apprenti.e.s en termes de rôle (Losa \& Filliettaz, 2018), appréhender leurs conflits, ambiguïté et surcharge de rôle peut s'avérer fort pertinent. Par ailleurs, la problématique des tensions de rôle en lien notamment avec les personnes en formation a été reprise en termes de légitimité des tâches à effectuer (Sacco, 2020).

\subsubsection{Les tâches illégitimes}

Les tâches illégitimes telles que définies par Semmer et al. $(2010,2015)$ sont des tâches «qui ne sont pas conformes à ce que l'on peut attendre d'une personne dans le cadre de son rôle ${ }^{5}$ (Semmer et al., 2015, p.33). En reprenant l'approche des tensions de rôle, les tâches illégitimes illustrent une sous-catégorie des conflits personne-rôle : il s'agit en effet de tâches perçues comme problématiques car incompatibles entre les valeurs de l'individu et le rôle défini par la tâche. Plus précisément, l'illégitimité est due aux attentes de rôle inappropriées compte tenu de la position professionnelle de la personne (Semmer et al., 2015). Les tâches illégitimes peuvent être non raisonnables ou non nécessaires (Sacco, 2020). Les tâches non raisonnables sont « des activités qu'il n'est pas adéquat d'exiger d'un individu [...] qui ne correspondent pas du tout aux attentes liées à la profession » (Sacco, 2020). C'est le cas par exemple d'un·e stagiaire universitaire ayant comme tâche de préparer le café aux collègues. Les tâches non nécessaires « ne devraient même pas exister. Certaines n'ont pas de sens, d'autres pourraient être évitées. Ou alors, il devrait être possible de les effectuer moyennant moins d'efforts. » (Sacco, 2020). Ainsi, demander par exemple à un·e employée de découper des boîtes en carton qui seront ensuite détruites est une tâche non nécessaire. Remarquons que les tâches illégitimes reflètent une certaine subjectivité des individus devant les effectuer: en effet, une tâche peut être considérée normale dans un certain cadre de travail alors qu'elle peut devenir illégitime dans un autre contexte professionnel. Par ailleurs, certaines tâches considérées ingrates font partie

\footnotetext{
${ }^{5}$ Traduction personnelle. Texte original : « tasks that do not conform to what can appropriately be expected from someone in terms of his or her role [...]» (Semmer et al., 2015, p.33).
} 
du métier ; leur appréciation dépend donc davantage de la fréquence et de la répartition entre collègues que de la tâche en elle-même.

Les tensions de rôle, englobant également la perception d'effectuer des tâches illégitimes, sont considérées dans la littérature comme des facteurs de stress dans le cadre professionnel. Cela signifie qu'elles peuvent expliquer certaines résultantes telles que l'insatisfaction au travail, mais aussi l'engagement ou l'intention de résilier le contrat de travail (Björk et al., 2013; Eatough et al., 2016 ; Semmer et al., 2010).

Comme suggéré auparavant, les tensions de rôle peuvent être assimilées à des caractéristiques définissant en partie la qualité en FP. Ainsi, considérer les liens entre tensions de rôle et certaines résultantes telles que la satisfaction ou l'engagement correspond en partie à considérer ces liens entre la qualité de la FP et les mêmes résultantes. Des études spécifiques en FP (Berweger et al., 2013 ; Stalder, 2003) ont en ce sens étudié les liens entre les perceptions de la qualité et certaines conséquences (engagement, satisfaction). Il a ainsi été démontré que plus les apprenti·e.s perçoivent leur formation comme étant de bonne qualité, plus elles et ils sont satisfait·e.s et engagé·e·s (Wenger et al., 2021). En appréhendant les tensions de rôle comme une caractéristique - négative - de la qualité en FP, il serait donc possible de suggérer des liens analogues avec certaines conséquences de la qualité. Par ailleurs, ces derniers liens ont déjà été étudiés et validés dans le contexte professionnel.

\subsection{Les résultats des tensions de rôle caractérisant la qualité en FP : auto-efficacité, engagement, satisfaction et intention de résilier le contrat}

Ces diverses tensions des rôles dans le cadre professionnel ont des conséquences multiples. Les études d'Alves et al. (2010a, 2010b) s'intéressent, dans le cadre de l'apprentissage en alternance en France, à l'impact des tensions de rôle (au sein de l'école et de l'entreprise formatrice, mais également entre les deux lieux) sur la satisfaction dans l'apprentissage, l'engagement et l'intention de rester dans l'entreprise après la formation. En ce sens, les auteur·e $\cdot s$ suggèrent que l'ambiguïté de rôle, tant au travail qu'à l'école, entretient un lien négatif avec la satisfaction qui, à son tour, est liée à l'engagement et à l'intention de rester dans l'entreprise formatrice (Alves et al., 2010b).

De plus, les méta-analyses des études concernant les tensions de rôle dans le cadre professionnel (Fisher \& Gitelson, 1983 ; Örtqvist \& Wincent, 2006) ont mis en évidence des liens entre l'ambiguïté, les conflits et la surcharge de rôle comme facteurs de stress et l'insatisfaction au travail, la baisse d'engagement professionnel ainsi que l'intention de résilier le contrat de travail. Plus précisément, chacune des facettes des tensions de rôle a des conséquences négatives sur des aspects-clés du bien-être au travail (Örtqvist \& Wincent, 2006).

L'approche des tâches illégitimes a quant à elle montré que ces dernières seraient en partie la cause d'une baisse de la confiance en soi, mais aussi de l'insatisfaction au travail (Eatough et al., 2016; Semmer et al., 2015). Ces résultats sont cohérents avec ce qui a été indiqué auparavant, sachant que les tâches illégitimes peuvent être considérées comme un cas spécifique des conflits de rôle ; ainsi, la perception d'effectuer des tâches non nécessaires ou non raisonnables semble être un facteur de stress au même titre que les tensions de rôle, provoquant par conséquent les mêmes effets négatifs que ces dernières.

En ce qui concerne le sentiment d'efficacité personnelle (Bandura, 1997), cette croyance serait négativement associée aux tensions de rôle (Beauchamp \& Bray, 2001; Caesens \& Stinglhamber, 2014 ; Yu et al., 2015) ; plus précisément, lorsqu'un individu endosse des rôles perçus comme ambivalents, cette croyance peut exercer une fonction médiatrice du lien entre les tensions de rôle et d'éventuelles conséquences telles que l'insatisfaction au travail 
(Dasgupta, 2012). Autrement dit, les tensions de rôle influencent le sentiment d'efficacité, luimême étant directement prédicteur de résultantes comme la satisfaction.

Il est donc intéressant d'observer que des facteurs de stress au travail tels que les tensions de rôle peuvent impacter négativement le bien-être des employé·e·s.

\section{Objectifs, questions de recherche et modèle hypothétique}

À partir de ces éléments de réflexion théoriques, les questions de recherche suivantes ont été émises :

1. Est-ce possible de distinguer plusieurs aspects des tensions de rôle en tant que caractéristiques de la qualité perçue ? Si oui, lesquels ?

2. Dans quelle mesure les perceptions des tensions de rôle sont-elles explicatives des conséquences de la qualité (engagement scolaire et professionnel, sentiment d'efficacité personnelle scolaire et professionnel, satisfaction de vie et intention de résilier le contrat d'apprentissage) ?

La figure 1 montre les liens qui seront testés : à partir des éléments concernant les tensions de rôle caractérisant la qualité de la FPI selon les apprenti·e·s, l'idée est d'observer s'il existe un lien avec les résultantes de cette qualité. Le sentiment d'efficacité personnelle (scolaire et professionnelle), l'engagement (scolaire et professionnel), la satisfaction de vie et l'intention de résilier le contrat d'apprentissage sont donc proposées comme conséquences des caractéristiques de la qualité. Plus précisément, le sentiment d'efficacité personnelle intègre le modèle en tant que médiateur du lien entre les caractéristiques de la qualité et les deux formes d'engagement (Caesens \& Stinglhamber, 2014 ; Yu et al., 2015). Par ailleurs, l'engagement devrait expliquer la satisfaction de vie qui, à son tour, expliquerait l'intention de résilier le contrat d'apprentissage: en effet, la satisfaction expliquerait, plus directement que l'engagement, le fait de vouloir résilier le contrat d'apprentissage (Alarcon \& Edwards 2011 ; Tett \& Meyer, 1993).

\section{Figure 1}

Liens postulés entre les tensions de rôle et leurs conséquences

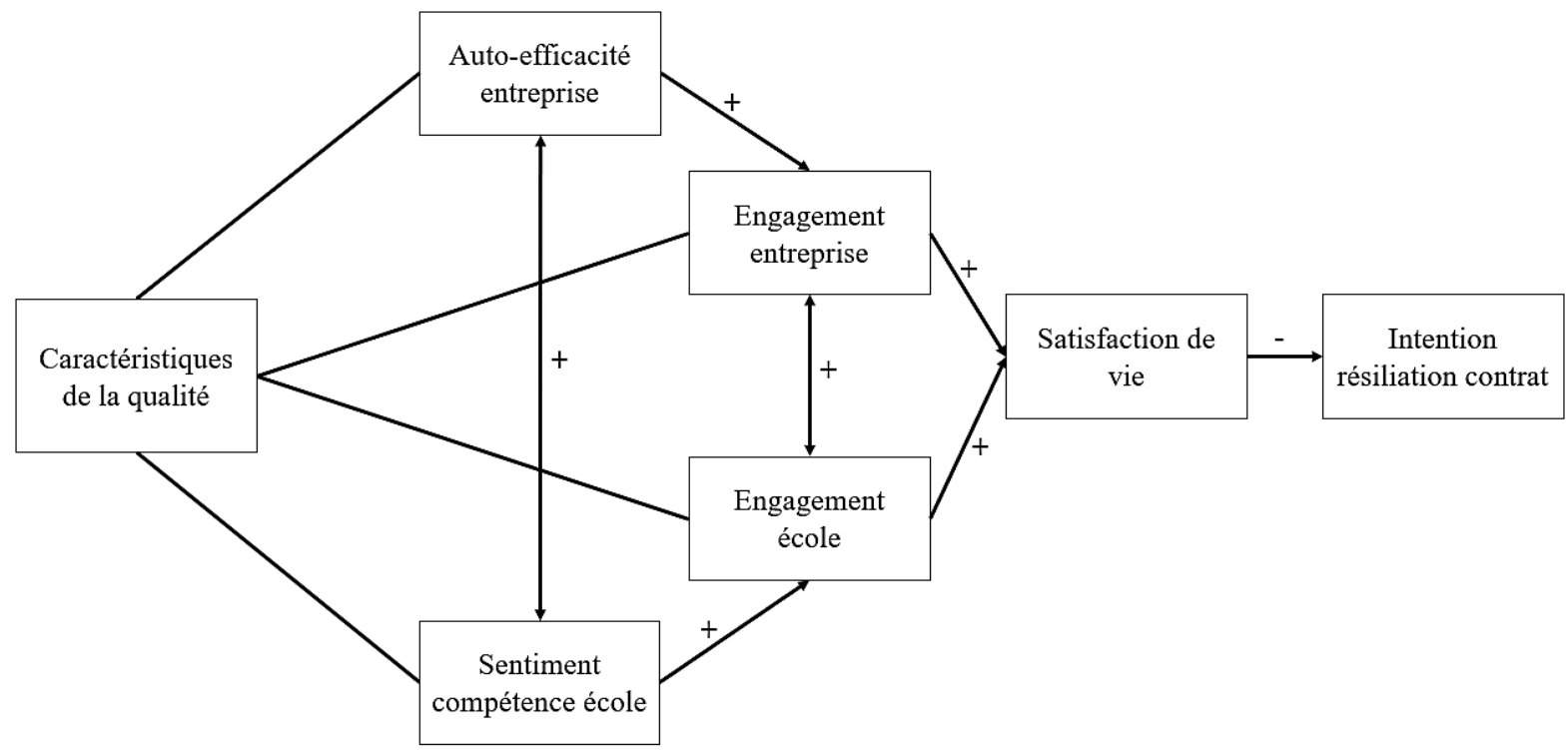

Figure 1

Postulated links between role stress and their consequences 
Dans un premier temps, des analyses factorielles exploratoires permettront la création de facteurs distincts faisant référence aux caractéristiques de la qualité perçue par les apprenti·e·s ; en ce sens, le modèle illustré à la figure 1 sera détaillé suite à la découverte des aspects descriptifs des tensions de rôle. Ensuite, des analyses de cheminement permettront de tester les liens postulés entre les caractéristiques de la qualité liées aux tensions de rôle et diverses conséquences de ces dernières (sentiment d'auto-efficacité et engagement scolaires et professionnels, satisfaction et intention de résilier le contrat d'apprentissage).

\section{Méthode}

\subsection{L'étude dans son contexte}

Cette étude s'inscrit dans un projet plus large, visant à investiguer la notion de qualité en FPI à partir des diverses perceptions des enseignant.e.s, personnes formatrices en entreprise et apprenti-e.s dans les quatre champs professionnels précités. Le projet a débuté par une étude exploratoire par questionnaire (questions ouvertes à réponses courtes) auprès d'apprenti.e.s (Berger et al., 2019b ; Gross et al., 2020) dont le but était de mettre en évidence les aspects caractérisant une formation de plus ou moins bonne qualité. A partir de ces résultats, des entretiens collectifs avec des groupes d'enseignant.e.s, personnes formatrices et apprenti.e.s ont permis d'affiner et de confirmer les éléments caractérisant la qualité de la FPI. Parmi ces éléments, nous avons notamment identifié la thématique des tensions de rôle en tant que caractéristique négative de la qualité (Wenger, sous presse). L'analyse thématique des entretiens a donné lieu à un questionnaire reprenant les thèmes identifiés dans les différents discours $^{6}$, questionnaire qui a ensuite été soumis à un échantillon d'apprenti·e.s en FPI. Les réponses à une partie des items et échelles sont utilisées dans le cadre de cette étude. Plus précisément, parmi les facteurs caractérisant la qualité en FPI, les analyses portent ici sur ceux rendant compte du vécu des apprenti·e·s à travers des tensions de rôle.

\subsection{Participant $\cdot e \cdot s$}

Les personnes ayant participé à l'étude sont des apprenti-e.s en FPI duale ${ }^{7}(N=336 ; 60.7 \%$ femmes) : 197 employé·e.s de commerce, soit 58.6\% de l'échantillon et 139 gestionnaires du commerce de détail, soit $41.4 \%$ de l'échantillon. Les apprenti.e.s étaient reparti.e.s entre la $1^{\text {ère }}$ $(n=149 ; 44.3 \%)$, la $2^{\mathrm{e}}(n=101 ; 30.1 \%)$ et la $3^{\mathrm{e}}$ année $(n=86 ; 25.6 \%)$ d'apprentissage. L'âge moyen est de 18.8 ans $(E T=3.47)$. Il s'agissait de 22 classes d'apprenti·e·s dans deux écoles professionnelles de deux cantons Suisses romands.

\subsection{Procédure}

Un questionnaire au format papier a été complété par les apprenti.e.s sur une période de cours, soit environ 45 minutes. Un membre de l'équipe de recherche était présent lors des passations du questionnaire afin d'assurer une certaine standardisation. La participation à l'étude était facultative, anonyme, non rémunérée et n'a pas été présentée comme une activité de l'école. En outre, le plan de recherche et la procédure de passation ont été approuvés par le Fonds national suisse de la recherche scientifique (FNS) et les cantons dans lesquels l'enquête a été réalisée.

\footnotetext{
${ }^{6}$ En guise d'exemple, d'autres facteurs identifiés comme caractérisant la qualité de la FP sont : l'articulation entre l'école et l'entreprise, les compétences des enseignant·e-s et personnes formatrices ou encore l'organisation curriculaire de la FP.

${ }^{7}$ La FPI peut être débutée dès la scolarité obligatoire achevée, scolarité qui se termine généralement à 15 ans.
} 


\subsection{Instruments}

Afin de tester le modèle postulé, plusieurs items issus du questionnaire ont été utilisés : d'une part, il s'agit d'items concernant les caractéristiques de la qualité de la formation perçue par les apprenti.e.s (en termes de tensions de rôle); d'autre part, ce sont des instruments scientifiquement validés qui ont été exploités en tant que mesures des conséquences de la qualité perçue.

\subsubsection{Les instruments concernant les tensions de rôle}

Les items faisant référence à la qualité de la formation des apprenti·e·s ont été développés à partir des thématiques identifiées lors d'entretiens collectifs (Berger et al., 2020). Ainsi, le thème des tâches et responsabilités des apprenti-e.s a permis de développer 16 items distincts, répartis en trois sous-thématiques : stress de rôles (ex : "A l'école, les enseignant·e's me traitent comme un·e enfant »; 6 items), diversité des tâches (ex : «Au travail, j'effectue des tâches variées »; 4 items) et responsabilisation des apprenti.e.s (ex : «Au travail, mes collègues me confient des tâches qu'ils et elles ne veulent pas faire »; 6 items). La première partie des résultats illustrera à ce propos l'analyse factorielle exploratoire effectuée dans le but d'extraire les facteurs caractérisant la qualité de la formation en termes de tensions de rôle des apprenti·e.s.

\subsubsection{Les instruments concernant les conséquences de la qualité perçue}

Concernant les variables à expliquer dans le modèle postulé, les diverses dimensions utilisées dans le questionnaire sont issues des échelles suivantes :

- Sentiment de compétence à l'école (ex : « Je crois être un·e bon·ne étudiant·e »; 4 items ${ }^{8}$; Losier et al., 1993), soit le fait de se percevoir comme étant compétent·e dans les tâches scolaires ;

- Sentiment d'efficacité personnelle en entreprise (ex : « Je me sens à la hauteur de la plupart des exigences professionnelles »; 7 items ; Occupational Self-Efficacy Scale ; Rigotti et al., 2008), que les auteur·e·s définissent comme «la compétence ressentie par une personne quant à sa capacité à accomplir les tâches concernées dans son travail ${ }^{9}$ » (Rigotti et al., 2008, p. 239);

- Engagement à l'école (ex : «J'écoute très attentivement durant les cours »; 12 items), initialement composé de trois sous-dimensions : engagement comportemental, émotionnel (Skinner et al., 2008) et cognitif (Simons et al., 2004). Il s'agit du fait de s'investir dans les tâches scolaires ;

- Engagement en entreprise (ex : «Je suis passionné-e par mon travail »), composé de trois sous-dimensions : vigueur, dévouement et absorption (3 items ; UWES ; Schaufeli et al., 2006) ;

- Intention de résilier le contrat d'apprentissage (ex : « Je pense à arrêter cet apprentissage pour changer de métier »; 3 items ; Berger et al., 2019b), à savoir l'intention de quitter la formation actuellement suivie pour une autre formation ou une autre entreprise formatrice ;

\footnotetext{
${ }^{8}$ Un item a finalement été supprimé à la suite des analyses factorielles confirmatoires, pour cause de saturation trop faible.

${ }^{9}$ Traduction personnelle. Texte original : «[... the competence that a person feels concerning the ability to successfully fulfill the tasks involved in his or her job. » (Rigotti et al., 2008, p.239).
} 
- Satisfaction de vie d'apprenti·e (ex : «Je suis satisfait·e de ma vie d'apprenti·e »; 5 items ; Alves et al., 2010b), échelle adaptée à partir de l'appréciation globale sur la qualité de vie (Diener et al., 1985).

Pour tous les items, l'échelle de réponse s'étendait de $1=$ Ne me correspond pas du tout à $6=$ Me correspond tout à fait, à l'exception de l'Engagement en entreprise (de $0=$ Jamais à $6=$ Toujours) et la Satisfaction de vie d'apprenti $\cdot e$ (de $1=$ Fortement en désaccord à $7=$ Fortement en accord).

\section{Résultats}

Les résultats sont présentés en deux parties : d'abord, l'analyse factorielle exploratoire des items sur la qualité de la formation dans le but de faire ressortir des dimensions liées aux tensions de rôle ; ensuite, à partir des facteurs identifiés, des analyses de cheminement ont été réalisées, comme postulés dans la figure 1.

\subsection{Analyse factorielle exploratoire}

En effectuant une analyse factorielle exploratoire avec le logiciel SPSS (avec factorisation en axes principaux et rotation Oblimin directe), la solution factorielle suivante a été jugée satisfaisante (variance totale cumulée : $64.67 \%$; voir tableau 1) : elle distingue quatre facteurs, deux faisant référence aux tâches à effectuer en entreprise et deux autres concernant les tensions de rôle chez les apprenti.e.s. Ainsi, pour répondre à la première question de recherche, les quatre facteurs suivants ont été extraits : le Facteur 1 correspond à l'impression de Devoir effectuer des tâches ingrates, soit effectuer le travail que les collègues ne veulent pas faire ; le Facteur 2 concerne la Diversité des tâches, à savoir la possibilité d'effectuer des tâches variées en entreprise ; le Facteur 3, Être considéré·e comme un·e employé·e, faisant référence à la perception d'effectuer des tâches au travail que font habituellement les employé·e·s et pas les apprenti.e.s ; le Facteur 4 est le sentiment d'Être traité.e comme une personne adulte et reprend la dichotomie entre le fait d'être encore traité.e comme un·e enfant $v s$ un·e adulte par les enseignant.e.s en école professionnelle.

Tableau 1

Résultats de l'analyse factorielle exploratoire des items évaluant les tensions de rôle

\begin{tabular}{|c|c|c|c|c|}
\hline & \multicolumn{4}{|c|}{ Facteur } \\
\hline & $\begin{array}{l}\text { Tâches } \\
\text { ingrates }\end{array}$ & $\begin{array}{l}\text { Diversité } \\
\text { tâches }\end{array}$ & $\begin{array}{c}\text { Etre } \\
\text { considéré·e } \\
\text { un·e employé·e }\end{array}$ & $\begin{array}{l}\text { Être traité·e } \\
\text { comme un·e } \\
\text { adulte }\end{array}$ \\
\hline $\begin{array}{l}\text { Être apprenti·e, c'est devoir faire les « tâches ingrates » que } \\
\text { les collègues ne veulent pas faire. }\end{array}$ & .55 & & & \\
\hline $\begin{array}{l}\text { Au travail, mes collègues me confient des tâches qu'ils et } \\
\text { elles ne veulent pas faire. }\end{array}$ & .73 & & & \\
\hline Mes collègues profitent de mon statut d'apprenti $\cdot e$ pour me & .80 & & & \\
\hline
\end{tabular}

faire faire des activités désagréables

Au travail, j'effectue des tâches variées. $\quad .86$

Au travail, je fais toujours la même chose. $\quad-.64$

Au travail, je vois beaucoup d'activités différentes. $\quad .86$

Au travail, mes tâches sont répétitives. $\quad-.61$

Au travail, je me sens comme un·e employé·e.

Au travail, je suis traité·e comme un·e employé·e plutôt que $\quad-.86$

comme un·e apprenti·e.

Au travail, on me donne des responsabilités.

À l'école, je me sens traité·e comme un·e adulte responsable. 
À l'école, les enseignant·e $\cdot s$ me traitent comme un·e enfant.

Le travail me permet d'être une personne responsable.

\section{Table 1}

Results of the exploratory factor analysis of items assessing role stress

Deux items ayant des saturations inférieures à .30 ont été supprimés ${ }^{10}:$ (a) Mon formateur ou ma formatrice considère que je dois encore apprendre le métier ; (b) Les responsabilités que j'ai au travail sont adaptées à mes connaissances.

\subsection{Analyses de cheminement}

A la suite des résultats de l'analyse factorielle, des analyses de cheminement ont été effectuées par le biais du logiciel statistique Mplus (version 7.11 ; Muthén \& Muthén, 2020). Cela a permis de tester les liens postulés entre les perceptions de la qualité et leurs résultantes (voir figure 2), en intégrant les quatre dimensions de la qualité en variables explicatives du modèle.

\section{Figure 2}

Modèle théorique développé suite à l'analyse factorielle - liens postulés entre les tensions de rôle et leurs conséquences

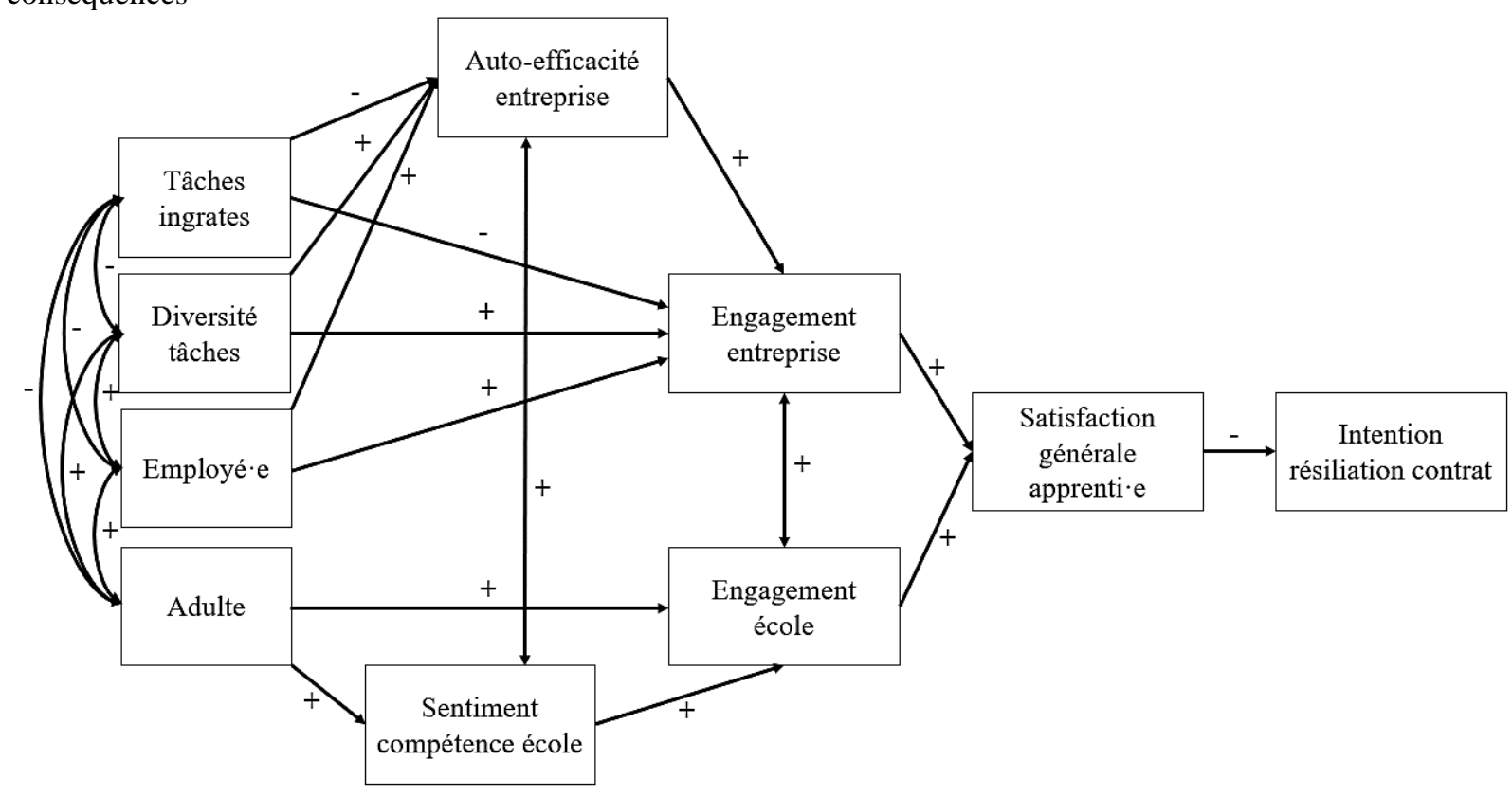

Figure 2

Theoretical model developed following factor analysis - postulated links between role stress and their consequences

Le tableau 2 présente les statistiques descriptives et les corrélations entre les variables. Les alphas de Cronbach (indiqués entre parenthèses dans le tableau) sont satisfaisants pour toutes les échelles, sauf celle du sentiment de compétence à l'école qui présente un alpha acceptable

${ }^{10}$ Ce seuil étant lié à la taille de l'échantillon et se situant donc à .30 pour un échantillon de 350 individus (Hair et al., 2014). 
et d'être traité.e comme une personne adulte dont la consistance interne est insatisfaisante. Toutefois, la validité de ce dernier score est soutenue par l'analyse factorielle. Quant au sentiment de compétence à l'école, ce résultat pourrait être dû à un certain manque de compréhension des items au vu de l'origine québécoise de l'instrument.

Par la suite, les différents liens postulés dans la figure 2 ont été testés par le biais d'une analyse de cheminements.

\section{Tableau 2}

Statistiques descriptives et corrélations entre les variables

\begin{tabular}{|c|c|c|c|c|c|c|c|c|c|c|}
\hline Echelle & 1. & 2. & 3. & 4. & 5. & 6. & 7. & 8. & 9. & 10. \\
\hline 1. Tâches ingrates & $(.80)$ & & & & & & & & & \\
\hline 2. Diversité tâches & -.48 & $(.84)$ & & & & & & & & \\
\hline 3. Adulte & -.12 & .21 & $(.54)$ & & & & & & & \\
\hline 4. Employé·e & -.29 & .27 & .19 & $(.71)$ & & & & & & \\
\hline 5. Sentiment compétence école & -.06 & .12 & .25 & .14 & $(.61)$ & & & & & \\
\hline 6. Engagement école & -.11 & .23 & .44 & .11 & .49 & $(.86)$ & & & & \\
\hline 7. Engagement entreprise & -.42 & .54 & .27 & .28 & .22 & .43 & $(.83)$ & & & \\
\hline 8. Auto-efficacité entreprise & -.23 & .23 & .17 & .36 & .24 & .18 & .43 & $(.83)$ & & \\
\hline 9. Satisfaction générale & -.37 & .45 & .30 & .26 & .18 & .32 & .61 & .38 & $(.90)$ & \\
\hline 10. Intention résiliation contrat & .26 & -.27 & -.10 & -.07 & -.05 & -.02 & -.33 & -.17 & -.38 & $(.82)$ \\
\hline Moyenne & 2.73 & 4.03 & 4.84 & 4.71 & 4.23 & 4.27 & 4.05 & 4.42 & 4.58 & 1.65 \\
\hline Ecart-type & 1.30 & 1.20 & .83 & .98 & .93 & .81 & 1.26 & .81 & 1.39 & 1.11 \\
\hline
\end{tabular}

Entre parenthèses sont indiqués les alphas de Cronbach pour chacun des scores.

N.B. les corrélations $\geq|.12|$ sont significatives au seuil de .05 ; les corrélations $\geq|.17|$ sont significatives au seuil de .01 ; les corrélations $\geq|.21|$ sont significatives au seuil de .001 .

\section{Table 2}

Descriptive statistics and correlations between variables

\subsubsection{Analyses de cheminement : test du modèle}

Les résultats du modèle postulé sont indiqués à la figure 3. Bien que globalement satisfaisant, ce modèle présente des indices d'adéquation à peine suffisants (voir tableau 3), ce qui a justifié la mise en place d'un modèle incluant l'ajout de liens significatifs suggérés par les indices de modification offerts par Mplus. Ce modèle révisé présente de meilleurs indices du fit $(\triangle C F I=$ $.04 ; \triangle T L I=.07 ; \triangle R M S E A=.02 ;$ voir tableau 3 ). 
Figure 3

Résultats du modèle postulé des liens entre les tensions de rôle et leurs conséquences

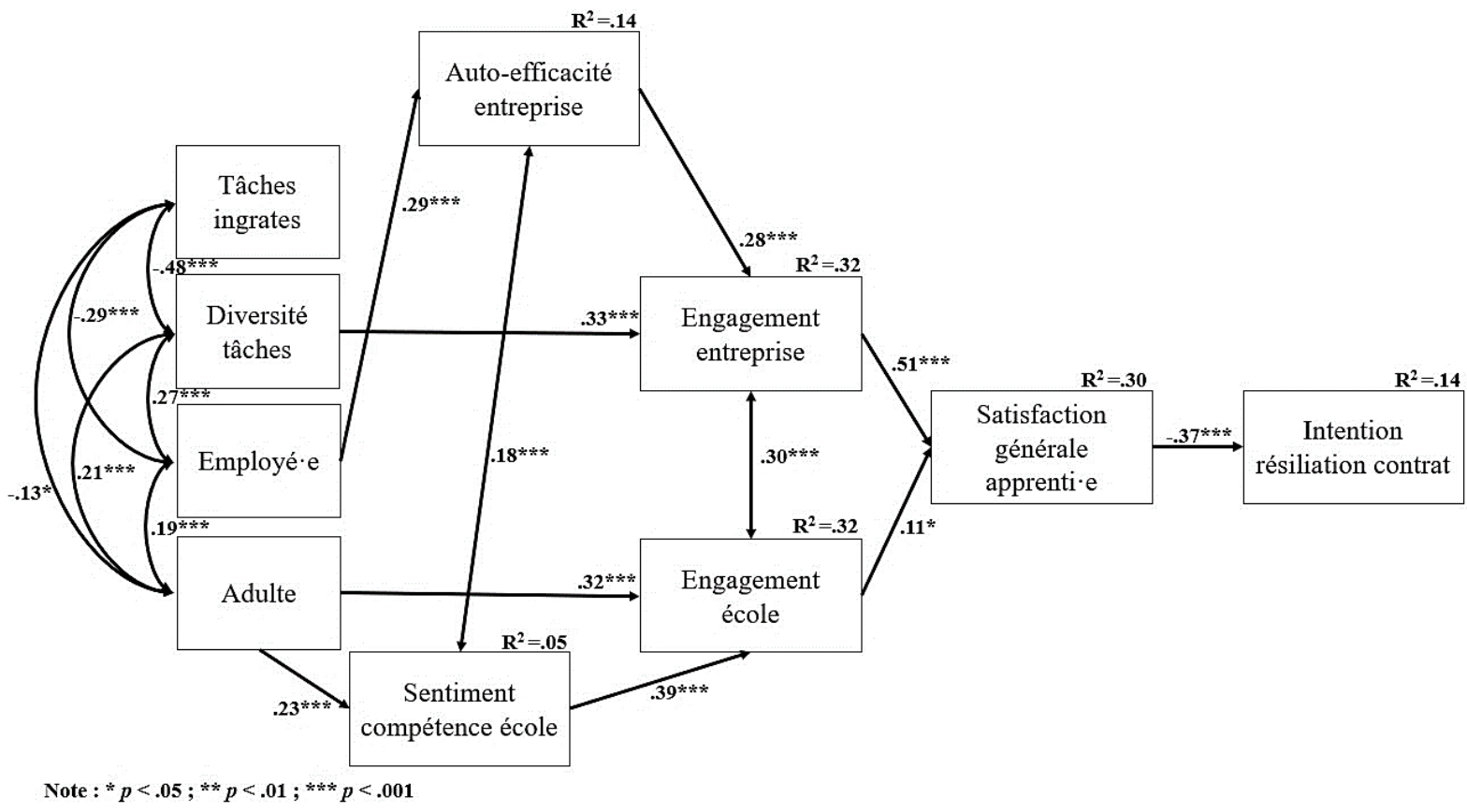

Figure 3

Results of the postulated model of links between role stress and their consequences

Tableau 3

Indices d'adéquation des modèles testés $(N=336)$

\begin{tabular}{lcccccccc}
\hline & $\chi^{2}$ & $\mathrm{df}$ & $\chi^{2} / \mathrm{df}$ & \multicolumn{4}{c}{ RMSEA CFI TLI SRMR } \\
\hline 1. Modèle postulé & 73.51 & 24 & 3.06 & $<.001$ & .08 & .90 & .83 & .08 \\
2. Modèle révisé & 54.61 & 25 & 2.18 & $<.001$ & .06 & .94 & .90 & .08
\end{tabular}

Table 3

Fit indices of the tested models $(\mathrm{N}=336)$

La figure 4 illustre les résultats schématisés du modèle révisé : comme dans le modèle théorique, le lien entre la perception d'être traité $\cdot e$ comme un'e employé $\cdot e$ et l'engagement en entreprise est intégralement médié par le sentiment d'efficacité personnelle, tandis que pour les deux autres facteurs de la qualité faisant référence à l'entreprise, il n'y a pas de médiation. Le sentiment de compétence en école, quant à lui, a un effet médiateur partiel du lien entre la perception d'être traité'e comme une personne adulte et l'engagement à l'école. Par ailleurs, l'engagement en entreprise est aussi expliqué par la perception d'effectuer des tâches variées ; seule la perception d'effectuer des tâches ingrates en entreprise n'est finalement pas explicative de l'engagement en entreprise. En outre, la satisfaction est expliquée directement par trois des quatre dimensions de la qualité prise en compte dans cette étude. Effectuer des tâches ingrates explique négativement la satisfaction, signifiant que plus les apprenti.e.s estiment devoir effectuer le «sale boulot» en entreprise, moins elles et ils se considèrent satisfait.e.s de leur vie d'apprenti·e $\cdot$. La diversité des tâches est positivement associée à la satisfaction, ce qui se traduit par le fait que plus les apprenti·e·s ont la possibilité d'effectuer des tâches diversifiées, plus leur satisfaction est élevée. Il est par ailleurs intéressant d'observer que ce lien est 
partiellement médié par l'engagement en entreprise, lui-même explicatif de la satisfaction. Finalement, se sentir traité·e comme une personne adulte à l'école entretient aussi un lien positif avec la satisfaction des apprenti.e.s. Comme dans le modèle théorique, l'intention de résilier le contrat d'apprentissage est expliquée négativement par la satisfaction, ce qui signifie que plus les apprenti.e.s se sentent satisfait.e.s de leur vie, moins elles et ils auront l'intention de résilier leur contrat d'apprentissage. Remarquons aussi que dans ce modèle révisé, le lien précédemment présent entre l'engagement à l'école et la satisfaction n'est pas statistiquement significatif.

Les effets indirects entre les quatre facteurs caractérisant la qualité de la formation et l'intention de résilier le contrat d'apprentissage ont également été calculés (voir Annexe A) : ils ont permis de mettre en avant des liens indirects statistiquement significatifs pour trois des quatre facteurs (être considéré e comme un·e employé e ne présente pas d'effets significatifs). Aucun lien direct n'est cependant statistiquement significatif: les caractéristiques de la qualité n'expliquent donc pas directement l'intention de résilier le contrat d'apprentissage, mais le font à travers d'autres indicateurs, tels que la satisfaction de vie ou l'engagement.

Figure 4

Résultats du modèle révisé des liens entre les tensions de rôle et leurs conséquences

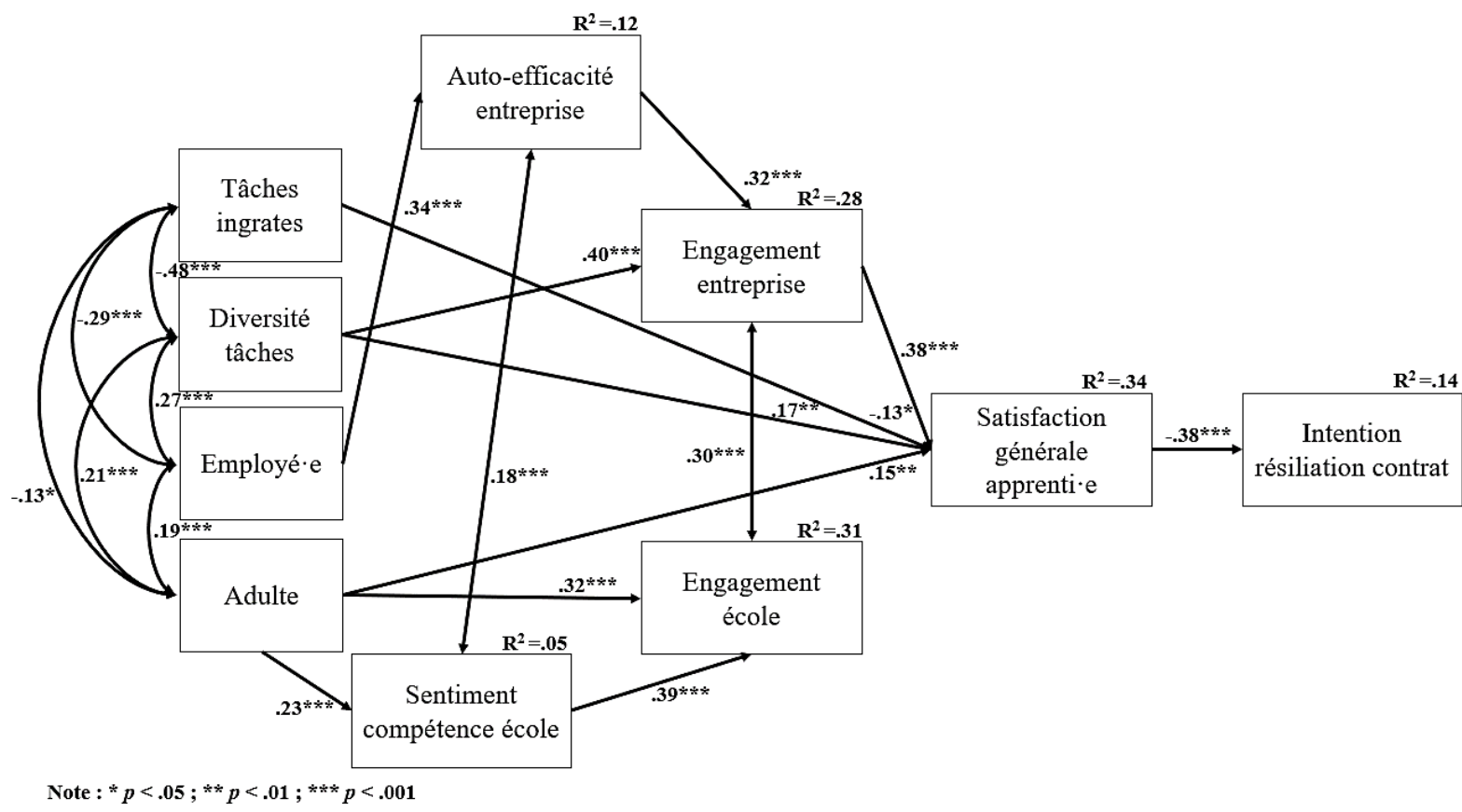

Figure 4

Results of the revised model of links between role stress and their consequences

\section{Discussion}

Cette étude visait à identifier les éventuels aspects des tensions de rôle en tant que caractéristiques de la qualité perçue et leur influence sur l'engagement et la satisfaction des apprenti·e.s. 


\subsection{Les aspects des tensions de rôle identifiés}

L'analyse factorielle exploratoire a permis d'identifier plusieurs aspects des tensions de rôle faisant référence autant à l'apprenti·e en tant que personne qu'aux tâches qu'elle ou il doit effectuer. Deux facteurs se réfèrent aux tâches au travail: Diversité des tâches et Devoir effectuer des tâches ingrates. Le premier s'approche de la dimension «Diversité » de l'IBAQ (Inventar zur betrieblichen Ausbildungsqualität) (Velten \& Schnitzler, 2012), alors que le deuxième correspond au concept de tâches illégitimes, notamment au niveau des tâches non raisonnables qui ne correspondent pas aux attentes liées au métier (Semmer et al., 2010, 2015). Les facteurs Être traité.e comme une personne adulte à l'école et Être considéré·e comme un·e employé e en entreprise peuvent quant à eux être associés à ce qui est nommé dans la littérature un conflit inter-rôle, soit l'existence de plusieurs rôles chez le même individu (Djabi \& Perrot, 2016). Ces deux facteurs reflètent une ambivalence au niveau identitaire : l'apprenti-e endosse des rôles multiples en fonction qu'elle ou il soit en école ou en entreprise, et parfois aussi au sein du même lieu de formation (par exemple se sentir à la fois enfant et adulte en école). Par conséquent, l'image de soi de l'apprenti-e change aussi, en lui demandant des efforts d'adaptation (Veillard \& Kouamé, 2014). Ainsi, Être traité.e comme une personne adulte à l'école évoque, sous un certain angle, une vision de l'école professionnelle comme institution paternaliste, qui amène à l'infantilisation des apprenti·e.s (Eckert \& Wenger, 1994). Sous un autre angle, ce facteur peut être aussi relié aux besoins typiques du passage de l'adolescence à l'âge adulte, soit la volonté d'acquérir davantage d'autonomie en réclamant son statut d'adulte, mais en fuyant parfois les responsabilités relatives à l'âge adulte en invoquant un statut d'enfant (Arnett, 2007). Le facteur Être considéré.e comme un·e employé.e reprend en partie les tensions entre former et produire (Moreau, 2006), deux logiques à la base de l'apprentissage par alternance: l'école étant l'institution vouée exclusivement à la formation, alors que l'entreprise formatrice se trouve tiraillée entre l'exigence de production et la formation des apprenti.e.s.

Les tensions de rôle identifiées dans la présente étude sont caractéristiques d'une formation par alternance. Les apprenti·e.s se trouvant parfois obligé·e.s d'assumer des rôles paradoxaux, ces tensions peuvent amener à des sentiments ambivalents et négatifs (Akkerman \& Bakker, 2012). De plus, les apprenti·e.s se retrouvent fréquemment seul·e.s dans la tâche de concilier leurs rôles multiples (Gurtner et al., 2018), alors que ce processus devrait être soutenu par le corps enseignant et les personnes formatrices (Sappa \& Aprea, 2018). L'approche du franchissement des frontières (Akkerman \& Bakker, 2012) permet à ce propos d'approfondir la réflexion entamée par la littérature sur les tensions de rôle. En effet, cette approche utilise les tensions de rôle (autant en termes identitaires que des tâches à effectuer) comme sources d'apprentissage potentielles. Autrement dit, le but n'est pas d'essayer de gommer la diversité, mais de la reconnaître et de l'intégrer dans les pratiques d'apprentissage autant en école qu'en entreprise. Ainsi, pour reprendre le concept de communautés de pratiques (Lave \& Wenger, 1991), les apprenti·e.s se trouvent à la périphérie des communautés de pratiques de l'école et de l'entreprise : elles et ils appartiennent simultanément aux deux communautés, mais pas non plus intégralement. En ce sens, les conflits inter-rôle pourraient être réduits en faisant vivre aux apprenti·e.s des expériences cohérentes entre école et entreprise (Sappa \& Aprea, 2018). Faire effectuer aux apprenti-e.s principalement des tâches ingrates ou répétitives ne concourt pas à construire cette cohérence. La suite de nos résultats, qui décrivent les liens entre tensions de rôle et leurs conséquences, permet de détailler davantage ce discours. 


\subsection{Les conséquences des tensions de rôle}

Les résultats des analyses nous ont aussi permis d'observer des liens statistiquement significatifs entre perceptions des tensions de rôle - en tant que caractéristiques de la qualité en FPI - et certaines conséquences. Ces résultats révèlent que les tensions de rôle expliquent des parts de variance non négligeables dans l'engagement en entreprise et en école, dans la satisfaction de vie et, finalement, l'intention de rompre le contrat d'apprentissage. Ainsi, les tensions de rôle constituent des aspects importants de la qualité perçue en FPI.

Les analyses révèlent que le sentiment d'effectuer des tâches ingrates présente un lien inverse à celui de la diversité des tâches sur la satisfaction : en ce sens, bien que ces deux dimensions de la qualité en entreprise ne soient pas les deux facettes d'une même problématique, il semblerait nécessaire, pour les personnes formatrices, de tenir compte de ces deux aspects distincts lors de l'organisation du travail des apprenti·e·s. Plus précisément, pour que les personnes en formation s'estiment satisfait·e $\cdot s$ de leur apprentissage et, indirectement, n'aient pas l'intention de résilier le contrat d'apprentissage, il est fondamental d'une part qu'elles se voient confier des tâches variées (Stalder \& Carigiet Reinhard, 2014) et, d'autre part, qu'elles ne se perçoivent pas comme seules préposées aux tâches ingrates dans l'entreprise (Sacco, 2020 ; Semmer et al., 2010, 2015).

Un autre résultat intéressant concerne le sentiment d'être considéré·e comme un·e employé·e. Ce ressenti, qui se voudrait révélateur d'une possible surcharge de travail, est lié à un plus haut sentiment d'efficacité personnelle en entreprise et indirectement à un plus haut engagement et une plus haute satisfaction. Ces résultats suggèrent que la valorisation des personnes en formation en leur confiant des responsabilités et des tâches en partie similaires à ce qu'effectuent les employé·e·s déjà formé·e.s, serait bénéfique sur plusieurs plans (Wenger, sous presse). Toutefois, les items de la présente étude ne laissent pas transparaître la face négative du concept, qui consisterait en une responsabilisation trop conséquente et des tâches trop difficiles à réaliser par les personnes en formation, menant vraisemblablement à une surcharge de rôle (Djabi \& Perrot, 2016 ; Djabi et al., 2019).

La perception d'être traité.e comme un·e adulte dans le cadre de l'école professionnelle importe de manières multiples : cette perception est liée directement au sentiment de compétence scolaire, à l'engagement dans les tâches scolaires (aussi indirectement via le sentiment de compétence scolaire) et à la satisfaction générale. Ainsi, l'école professionnelle, bien que jouant généralement un rôle mineur par rapport à l'entreprise formatrice (Alves et al., 2010b ; Sauli et al., 2019), aurait une importance dans la satisfaction et, de manière indirecte, également dans l'intention de résiliation du contrat d'apprentissage. Il serait donc important que l'école professionnelle et ses acteurs considèrent les apprenti $\cdot e \cdot s$ comme de jeunes adultes en formation plutôt que comme des élèves.

\section{Conclusion}

L'interprétation des résultats de cette étude présente plusieurs limites. Le processus de développement des items s'étant appuyé sur des analyses thématiques de deux études préalables, ces items n'ont pas été directement créés pour évaluer les aspects de tensions de rôle mis en évidence par la littérature. Si certains de ces aspects ont été identifiés par l'analyse factorielle, d'autres n'étaient pas présents dans les items. Les qualités psychométriques des scores sont globalement satisfaisantes ; toutefois, une prochaine étude permettra d'améliorer la validité des scores en couvrant un spectre plus large des tensions de rôle et en mesurant de manière plus exhaustive les dimensions concernées par la présente étude. Par ailleurs, les liens mis en évidence par les analyses de cheminement ne sont pas interprétables comme des liens 
de cause à effet étant donné le plan de recherche de type transversal appliqué. Ainsi serait-il pertinent de répliquer les résultats par l'utilisation d'un plan de recherche longitudinal.

Finalement, nous pouvons interroger le degré de généralisation des résultats : dans le cas de formation à des métiers différents, les mêmes conclusions pourraient-elles être tirées ? La suite de nos recherches devrait permettre de répondre à cette question par la considération de deux autres domaines professionnels que sont la coiffure et les soins de beauté ainsi que la construction. Nous formulons l'attente d'une réplication globale des résultats de la présente étude, tout en sachant que les diverses tensions de rôle se manifestent probablement de manière diverse selon les domaines professionnels.

Par la suite, il serait intéressant de comparer les deux champs professionnels afin d'observer s'il existe des différences tant par rapport aux perceptions de la qualité que des liens avec les résultats de cette dernière. De plus, et pour pallier à la limite mise en évidence auparavant, soit la construction successive des items relatifs aux tensions de rôle, il serait nécessaire de tester directement les dimensions liées aux tensions de rôle en tant que facteurs, c'est-à-dire de ne pas les créer a posteriori (suite notamment à une analyse factorielle). Cela permettrait de renforcer la puissance statistique des construits théoriques et des divers modèles testés. 


\section{Bibliographie}

Akkerman, S. F., \& Bakker, A. (2012). Crossing boundaries between school and work during apprenticeships. Vocations and learning, 5(2), 153-173. http://doi.org/10.1007/s12186$\underline{011-9073-6}$

Alarcon, G. M., \& Edwards, J. M. (2011). The relationship of engagement, job satisfaction and turnover intentions. Stress and Health, 27(3), e294-e298. https://doi.org/10.1002/smi.1365

Alves, S., Gosse, B., \& Sprimont, P. A. (2010a). Dimensions et conséquences de la satisfaction au travail des apprentis. Revue internationale de psychosociologie, 16(40), 161-180. https://doi.org/10.3917/rips.040.0161

Alves, S., Gosse, B., \& Sprimont, P. A. (2010b). Les apprentis de l'enseignement supérieur : de la satisfaction à l'engagement ? Management Avenir, (3), 35-51. https://doi.org/10.3917/mav.033.0035

Arnett, J. J. (2007). Emerging adulthood: What is it, and what is it good for? Child development perspectives, 1(2), 68-73. https://doi.org/10.1111/j.1750-8606.2007.00016.x

Bandura, A. (1997). Self-efficacy: The exercise of control. W. H. Freeman.

Beauchamp, M. R., \& Bray, S. R. (2001). Role ambiguity and role conflict within interdependent teams. Small group research, 32(2), 133-157. https://doi.org/10.1177\%2F104649640103200202

Berger, J.-L., Sauli, F., Wenger, M., \& Gross, V. (2019a). Evaluer la qualité d'une formation par les perceptions des participant·e.s : premiers résultats d'une recherche dans le contexte de la formation professionnelle initiale suisse. In C. Gremion, E. Sylvestre, \& $\mathrm{N}$. Younes (Eds.), Actes du 31ème Colloque scientifique international de l'ADMEEEurope : Entre normalisation, contrôle et développement formatif. Évaluations sources de synergies? (pp. 74-81). IFFP et CSE de l'Université de Lausanne.

Berger, J.-L., Wenger, M., \& Sauli, F. (2019b). How the perceived quality of in-company training matters: A study with apprentices in technical and retail occupations. In F. Marhuenda \& M. J. Chisvert-Tarazona (Eds.), Pedagogical concerns and market demands in VET. Proceedings of the 3rd Crossing Boundaries in VET conference, Vocational Education and Training Network (VETNET) (pp.187-194). https://doi.org/10.5281/zenodo.2641022

Berger, J.-L., Wenger, M., \& Sauli, F. (2020). La qualité de la formation professionnelle duale en Suisse. Education permanente, 223, 91-100.

Berweger, S., Krattenmacher, S., Salzmann, P., \& Schönenberger, S. (2013). Lernende im Spannungsfeld von Ausbildungserwartungen, Ausbildungsrealität und erfolgreicher Erstausbildung. Projektbericht [Les apprentis dans le champ de tension entre les attentes en matière de formation, la réalité de la formation et la réussite de la formation initiale] [Rapport de projet]. Pädagogische Hochschule St. Gallen, Institut Professionsforschung und Kompetenzentwicklung.

Billett, S. (2001). Learning through work: workplace affordances and individual engagement. Journal of workplace learning, 13(5), 209-214. https://doi.org/10.1108/EUM0000000005548

Billett, S. (2009). Modalités de participation au travail : la dualité constitutive de l'apprentissage par le travail. Travail et formation des adultes, 37-63. 
https://doi.org/10.3917/puf.duran.2009.01.0035

Björk, L., Bejerot, E., Jacobshagen, N., \& Härenstam, A. (2013). I shouldn't have to do this: Illegitimate tasks as a stressor in relation to organizational control and resource deficits. Work \& Stress, 27(3), 262-277. https://doi.org/10.1080/02678373.2013.818291

Bouchard, C., \& Plante, J. (2002). La qualité : mieux la définir pour mieux la mesurer. Les Cahiers du Service de pédagogie expérimentale, 11-12, 219-236.

Caesens, G., \& Stinglhamber, F. (2014). The relationship between perceived organizational support and work engagement: The role of self-efficacy and its outcomes. European Review of Applied Psychology, 64(5), 259-267. https://doi.org/10.1016/j.erap.2014.08.002

CSFO (2012). QualiCarte: Instruments à télécharger. Formationprof. https://www.berufsbildung.ch/dyn/7361.aspx

Dasgupta, P. (2012). Effect of Role Ambiguity, Conflict and Overload in Private Hospitals' Nurses' Burnout and Mediation Through Self Efficacy. Journal of Health Management, 14(4), 513-534. https://doi.org/10.1177\%2F0972063412468980

Diener E., Emmons R. A., Larsen R. J., \& Griffin S. (1985). The Satisfaction With Life Scale. Journal of Personality Assessement, 49, 71-76. https://doi.org/10.1207/s15327752jpa4901_13

Djabi, M., \& Perrot, S. (2016). Tensions de rôle : proposition d'une grille d'analyse. Management international/International Management/Gestiòn Internacional, 21(1), 140-148. https://doi.org/10.7202/1052503ar

Djabi, M., Perrot, S., Jeannerod-Dumouchel, N., \& Campoy, É. (2019). Proposition d'une nouvelle échelle de mesure multidimensionnelle des tensions de rôle au travail. Revue de gestion des ressources humaines, (2), 41-65. https://doi.org/10.3917/grhu.112.0041

Dubar, C. (1992). Formes identitaires et socialisation professionnelle. Revue française de sociologie, 33(4), 505-529. https://doi.org/10.2307/3322224

Dubs, R. (2006). Rapport d'expertise sur les questions concernant la formation professionnelle en Suisse. HEP.

Eatough, E. M., Meier, L. L., Igic, I., Elfering, A., Spector, P. E., \& Semmer, N. K. (2016). You want me to do what? Two daily diary studies of illegitimate tasks and employee well-being. Journal of organizational behavior, 37(1), 108-127.

https://doi.org/10.1002/job.2032

Eckert, P., \& Wenger, E. (1994). From school to work: an apprenticeship in institutional identity [Document de travail]. Learning and Identity Initiative, Institute for Research on Learning.

Fisher, C. D., \& Gitelson, R. (1983). A meta-analysis of the correlates of role conflict and ambiguity. Journal of applied psychology, 68(2), 320. https://doi.org/10.1037/0021$\underline{9010.68 .2 .320}$

Griffin, T. (2017). Are We All Speaking the Same Language? Understanding "Quality" in the VET Sector. Occasional Paper. National Centre for Vocational Education Research (NCVER).

Gross, V., Wenger, M., Sauli, F., \& Berger, J.-L (2020). Motivating styles in dual initial vocational education and training: Apprentices' perceptions of autonomy support and control. JOVACET, 3(1), 67-88. https://doi.org/10.14426/jovacet.v3i1.126 
Guile, D., \& Young, M. (2003). Transfer and transition in vocational education: Some theoretical considerations. In T. Tuomi-Gröhn \& Y. Engeström (Eds.), Between school and work: New perspectives on transfer and boundary-crossing (pp. 63-81). Emerald Group Publishing Limited.

Gurtner, J.-L., Furlan, N., \& Cattaneo, A. (2018). L'articulation des connaissances n'est pas la tâche des seul·e.s apprenti·e.s. In L. Bonoli, J.-L. Berger, \& N. Lamamra (Eds.), Enjeux de la formation professionnelle. Le «modèle » suisse sous la loupe (pp. 253-266). Seismo.

Hair, J. F., Black, W. C., Babin, B. J., Anderson, R. E., \& Tatham, R. L. (2014). Multivariate data analysis $\left(7^{\text {th }}\right.$ ed.). Pearson New International Edition.

Lave, J., \& Wenger, E. (1991). Situated learning: Legitimate peripheral participation. Cambridge University Press.

Losa, S. A., \& Filliettaz, L. (2018). Négocier sa légitimité d'apprenant-e à travers les contextes de formation:formation : exemples d'apprenti-es en formation duale. In L. Bonoli, J.-L. Berger, \& N. Lamamra (Eds), Enjeux de la formation professionnelle en Suisse. Le" modèle" suisse sous la loupe (pp. 294-322). Seismo.

Losier, G. F., Vallerand, R. J., \& Blais, M. R. (1993). Construction et validation de l'Échelle des Perceptions de Compétence dans les Domaines de Vie (EPCDV). Science et comportement, 23(1), 1-16.

Martineau, S., Portelance, L., \& Presseau, A. (2009). La socialisation au travail comme indicateur de développement professionnel : analyse des approches basées sur la mesure. Questions Vives. Recherches en éducation, 5(11), 243-258. https://doi.org/10.4000/questionsvives.614

Masdonati, J., Lamamra, N., Gay-des-Combes, B., \& De Puy, J. (2007). Les enjeux identitaires de la formation professionnelle duale en Suisse : un tableau en demi-teinte. Formation emploi. Revue française de sciences sociales, (100), 15-29. https://doi.org/10.4000/formationemploi.1253

Moreau, G. (2006). La scolarisation de l'apprentissage salarié. Les temps modernes, (3), 393419. https://doi.org/10.3917/ltm.637.0393

Muthén, L. K., \& Muthén, B. O. (2020). Mplus (version 7.11) [Logiciel informatique]. Muthén \& Muthén.

OFS (2017). Choix de formation au degré secondaire II, évolution. https://www.bfs.admin.ch/bfs/fr/home/statistiques/education-science/indicateursformation/systeme-formation-suisse/degre-formation/degre-secondaire-ii/formationsdegre-secondaire.assetdetail.2924593.html

OFS (2019). Indicateur de la législature : Taux de chômage des jeunes. https://www.bfs.admin.ch/bfs/fr/home/statistiques/themes-transversaux/monitoringprogramme-legislature/indicateurs/taux-chomage-jeunes.html

Örtqvist, D., \& Wincent, J. (2006). Prominent consequences of role stress: A meta-analytic review. International Journal of Stress Management, 13(4), 399-422.

Perrot, S. (2005, September). L'individu et l'organisation : une approche par le concept de socialisation. In Congrès de l'AGRH, Paris, 2005 (Publication-CD).

Plante, J., \& Bouchard, C. (1998). La qualité. Sa définition et sa mesure. Service social, 47(12), 27-61. https://doi.org/10.7202/706780ar 
Rauner, F. (2007). Kosten, Nutzen und Qualität der beruflichen Ausbildung. Institut Technik und Bildung.

Rigotti, T., Schyns, B., \& Mohr, G. (2008). A short version of the occupational self-efficacy scale: Structural and construct validity across five countries. Journal of Career Assessment, 16(2), 238-255. https://doi.org/10.1177\%2F1069072707305763

Rizzo, J. R., House, R. J., \& Lirtzman, S. I. (1970). Role conflict and ambiguity in complex organizations. Administrative science quarterly, 15(2), 150-163.

https://doi.org/10.2307/2391486

Royal, L., \& Brassard, A. (2010). Comprendre les tensions de rôles afin de mieux les prévenir et de contribuer au bien-être des employés. Gestion, 35(3), 27-33.

https://doi.org/10.3917/riges.353.0027

Sacco, F. (2020, 30 janvier). Les « tâches illégitimes », nouveau facteur de stress. HR Today. https://www.hrtoday.ch/fr/article/les-taches-illegitimes-nouveau-facteur-de-stress

Sappa, V., \& Aprea, C. (2018). L'apprentissage professionnel entre l'école et l'entreprise : défis, modèles et conceptions individuelles. In L. Bonoli, J.-L. Berger, \& N. Lamamra (Eds.), Enjeux de la formation professionnelle. Le "modèle » suisse sous la loupe (pp. 267-293). Seismo.

Sauli, F., Wenger, M., Gross, V. \& Berger, J.-L. (2019). The Quality of the Swiss Initial Vocational Education and Training System through Apprentices' Perception of the Connections between School and Training Company. In T. Deissinger, U. Hauschildt, P. Gonon, \& S. Fischer (Eds.), Contemporary Apprenticeship Reforms and Reconfigurations (pp. 20-23). Lit Verlag.

Schaufeli, W. B., Bakker, A. B., \& Salanova, M. (2006). The measurement of work engagement with a short questionnaire: A cross-national study. Educational and psychological measurement, 66(4), 701-716. https://doi.org/10.1177\%2F0013164405282471

SEFRI (2018). La formation professionnelle en Suisse. Faits et chiffres 2018. https://edudoc.ch/record/131680/files/Fakten_Zahlen_BB2018_fr.pdf

Semmer, N. K., Jacobshagen, N., Meier, L. L., Elfering, A., Beehr, T. A., Kälin, W., \& Tschan, F. (2015). Illegitimate tasks as a source of work stress. Work \& Stress, 29(1), 32-56. https://doi.org/10.1080/02678373.2014.1003996

Semmer, N. K., Tschan, F., Meier, L. L., Facchin, S., \& Jacobshagen, N. (2010). Illegitimate tasks and counterproductive work behavior. Applied Psychology, 59(1), 70-96. https://doi.org/10.1111/j.1464-0597.2009.00416.x

Simons, J., Dewitte, S., \& Lens, W. (2004). The role of different types of instrumentality in motivation, study strategies, and performance: Know why you learn, so you'll know what you learn! British Journal of Educational Psychology, 74(3), 343-360. https://doi.org/10.1348/0007099041552314

Skinner, E., Furrer, C., Marchand, G., \& Kindermann, T. (2008). Engagement and disaffection in the classroom: Part of a larger motivational dynamic? Journal of educational psychology, 100(4), 765. https://doi.org/10.1037/a0012840

Smith, E., \& Yasukawa, K. (2017). What makes a good VET teacher? Views of Australian VET teachers and students. International Journal of Training Research, 15(1), 23-40. http://doi.org/10.1080/14480220.2017.1355301 
Stalder, B. (2003). Ecole, travail, satisfaction des jeunes. In J. Amos, E. Böni, M. Donati, S. Hupka, \& T. Meyer (Eds.), Les deux premières années après l'école obligatoire. Résultats de l'étude longitudinale TREE (pp. 59-79). Office fédéral de la statistique.

Stalder, B. E., \& Carigiet Reinhard, T. (2014). Ausbildungsqualität aus Sicht von Lernenden und Betrieben in der Schweiz. Qualität in der Berufsbildung. Anspruch und Wirklichkeit. Berichte zur beruflichen Bildung, 97-115.

Tett, R. P., \& Meyer, J. P. (1993). Job satisfaction, organizational commitment, turnover intention, and turnover: path analyses based on meta-analytic findings. Personnel psychology, 46(2), 259-293. https://doi.org/10.1111/j.1744-6570.1993.tb00874.x

Tubre, T. C., \& Collins, J. M. (2000). Jackson and Schuler (1985) revisited: A meta-analysis of the relationships between role ambiguity, role conflict, and job performance. Journal of management, 26(1), 155-169. https://doi.org/10.1177\%2F014920630002600104

Veillard, L., \& Kouame, D. K. (2014). Conception collaborative d'un outil d'aide au transfert de connaissances enseignées en formation par alternance. Éducation et francophonie, 42(1), 42-64. https://doi.org/10.7202/1024564ar

Velten, S., \& Schnitzler, A. (2012). Inventar zur betrieblichen Ausbildungsqualität (IBAQ). Zeitschrift für Berufs-und Wirtschaftspädagogik, 108(4), 511-527.

Wenger, M. (sous presse). Des tensions de rôle chez des apprenti·e.s en formation en alternance en Suisse. Psychologie du travail et Ressources Humaines. l'Harmattan.

Wenger, M., Sauli, F., \& Berger, J.-L. (2021). Person-centred Approach to Assess Quality Perceptions of Role Stress in Dual Initial Vocational Education and Training Apprentices. In C. Nagele, B. E. Stalder, \& M. Weich (Eds.), Pathways in Vocational Education and Training and Lifelong Learning. Proceedings of the 4th Crossing Boundaries Conference in Vocational Education and Training, Muttenz and Bern online, 8. - 9. April (pp. 358-363). European Research Network on Vocational Education and Training, VETNET, University of Applied Sciences and Arts Northwestern Switzerland and Bern University of Teacher Education. http://doi.org/10.5281/zenodo.4603145

Wittek, L., \& Kvernbekk, T. (2011). On the problems of asking for a definition of quality in education. Scandinavian Journal of Educational Research, 55(6), 671-684. https://doi.org/10.1080/00313831.2011.594618

Yu, X., Wang, P., Zhai, X., Dai, H., \& Yang, Q. (2015). The effect of work stress on job burnout among teachers: The mediating role of self-efficacy. Social Indicators Research, 122(3), 701-708. http://doi.org/10.1007/s11205-014-0716-5 


\section{Annexes}

Annexe A

Tableau des effets directs, indirects et totaux du modèle révisé.

$\beta$ ES $p$

Adulte $\rightarrow$ Engagement école

Effet total

$.39 .06<.001$

Effet indirect Adulte $\rightarrow$ Sentiment compétence école $\rightarrow$ Engagement école

$.09 .02<.001$

Effet direct Adulte $\rightarrow$ Engagement école

$.31 .06<.001$

\section{Diversité tâches $\rightarrow$ Satisfaction}

Effet total

Effet indirect Diversité tâches $\rightarrow$ Engagement entreprise $\rightarrow$ Satisfaction

$.17 .03<.001$

Effet direct Diversité tâches $\rightarrow$ Satisfaction

$.19 .07<.01$

\section{Adulte $\rightarrow$ Intention résiliation contrat}

Effet total

Effet indirect Adulte $\rightarrow$ Satisfaction $\rightarrow$ Intention résiliation contrat

$-.10 .03<.001$

Effet direct Adulte $\rightarrow$ Intention résiliation contrat

$.02 .08 \quad .83$

\section{Diversité tâches $\rightarrow$ Intention résiliation contrat}

Effet total

$-.19 .06<.01$

Effet indirect Diversité tâches $\rightarrow$ Satisfaction $\rightarrow$ Intention résiliation contrat - $.09 .03<.001$

Effet direct Diversité tâches $\rightarrow$ Intention résiliation contrat $-.09 .06 \quad .14$

\section{Tâches ingrates $\rightarrow$ Intention résiliation contrat}

Effet total

$.14 .07<.05$

Effet indirect Tâches ingrates $\rightarrow$ Satisfaction $\rightarrow$ Intention résiliation contrat $.04 .02<.05$

Effet direct Tâches ingrates $\rightarrow$ Intention résiliation contrat .10 .06 .11

\section{Employé•e $\rightarrow$ Intention résiliation contrat}

Effet total $.05 .08 \quad .50$

Effet indirect Employé·e $\rightarrow$ Satisfaction $\rightarrow$ Intention résiliation contrat $-.03 .02 \quad .10$

Effet direct Employé·e $\rightarrow$ Intention résiliation contrat $.08 .07 \quad .27$

Table of direct, indirect and total effects of the revised model 